\title{
Tensor Based Blind Source Separation in Longitudinal Magnetic Resonance Imaging Analysis
}

\author{
Claudio Stamile ${ }^{1,2}$, François Cotton ${ }^{1,4}$, Dominique Sappey-Marinier ${ }^{1,5}$ and Sabine Van Huffel ${ }^{2,3}$
}

\begin{abstract}
Study of white matter (WM) fiber-bundles is a crucial challenge in the investigation of neurological diseases like multiple sclerosis (MS). In this activity, the amount of data to process is huge, and an automated approach to carry out this task is in order.

In this paper we show how tensor-based blind source separation (BSS) techniques can be successfully applied to model complex anatomical brain structures. More in detail, we show how through vector hankelization it is possible to formalize data extracted from WM fiber-bundles using a tensor model. Two main tensor factorization techniques, namely $\left(L_{r}, L_{r}, 1\right)$ block term decomposition (BTD) and canonical polyadic decomposition (CPD), were applied to the generated tensor. The information extracted from the factorization was then used to differentiate between sets of fibers, within the bundle, affected by the pathology and normal appearing fibers.

Performances of the proposed tensor-based model was evaluated on simulated data representing pathological effects of MS. Results show the capability of our tensor-based model to detect small pathological phenomena appearing along WM fibers.
\end{abstract}

\section{INTRODUCTION}

Application of Blind Source Separation (BSS) techniques in magnetic resonance imaging (MRI) for brain analysis constitutes an important field in biomedical image processing. Different BSS techniques were applied on MRI data like non-negative matrix factorization (NMF) [12]. Recently, BSS algorithms based on tensor models showed successful results in diverse applications like brain studies. For instance, they were successfully applied to event related potential (ERP) analysis [23] and tumor tissue type differentiation based on magnetic resonance spectroscopic imaging [4].

In this work we describe a first application of tensor factorization in the study of white matter (WM) fiber-bundles. More in detail, we show how tensor factorization could be a powerful tool to detect "pathological" regions in WM fiberbundles affected by multiple sclerosis (MS).

In the last decade, the interest in new methods to analyze WM fiber-bundles increased. More in detail, different findings [21], [3] show how the combination of diffusion tensor imaging (DTI) data with anatomical information obtained from tractography [11] constitutes an important tool to study the brain. Unfortunately, the current methods available in

\footnotetext{
${ }^{1}$ CREATIS UMR 5220 CNRS \& U1206 INSERM, Université de Lyon, Université Claude Bernard Lyon 1, INSA-Lyon, UJM-Saint Etienne, Villeurbanne, France

${ }^{2} \mathrm{KU}$ Leuven, Department of Electrical Engineering (ESAT), Division Stadius, Leuven, Belgium

3 imec, Leuven, Belgium

${ }^{4}$ Service de Radiologie, Centre Hospitalier Lyon-Sud, Hospices Civils de Lyon, Pierre-Bénite, France

${ }^{5}$ CERMEP - Imagerie du Vivant, Université de Lyon, Bron, France
}

literature do not allow to perform complex analysis of those data structures. More in detail, they simply analyze the mean signal profile changes along the fiber-bundles. If these approaches offer a good visual tool to analyze pathological effects along the fiber-bundles, they are not sensitive enough to detect the presence of small "abnormal" regions [15], especially when those changes are relatively small like in relapsing remitting (RR) form of MS. Moreover, these methods are not able to combine longitudinal DTI information. Furthermore, since these approaches perform just a global general mean profile analysis of the fiber-bundle, they do not allow to distinguish which sets of fibers within the bundle are affected by the pathology and which are not.

In order to improve the quality of the fiber-bundle analysis we introduce a new tensor-based model to perform such a task. The idea behind our method, is to stack the large amount of data extracted from a given fiber-bundle using a $3^{r d}$ order tensor. In this process we will use a well known technique called hankelization [1], to stack the signal in a tensor. The obtained tensor is then factorized to extract meaningful information to separate respectively fibers, within a bundle, affected by the pathological process and healthy fibers.

Canonical polyadic decomposition (CPD) and block term decomposition (BTD) are used and compared to analyze the effects that different types of tensor decomposition have on fiber-bundle differentiation.

The performance of the proposed tensor-based model was evaluated on simulated data representing pathological effects of MS. Results show the capability of our tensor-based model to distinguish between fibers affected by a longitudinal pathological process and fibers belonging to normal tissue.

This paper is structured as follows. In Section II, we provide a detailed description of our approach. In Section III, we present our experimental campaign. In Section IV, we show the performance of our method. In Section V, we discuss our results. Finally, in Section VI, we draw our conclusions.

\section{MATERIALS AND METHODS}

\section{A. Data acquisition protocol}

One healthy control (HC) subject (age: 24y) was included in this study. The subject underwent a weekly examination for a period of two months (8 time-points). The DTI image set consisted of the acquisition of 60 contiguous $2 \mathrm{~mm}$-thick slices parallel to the bi-commissural plane (AC-PC), and were acquired using a 2D Echo-Planar Imaging (EPI) sequence $\left(\right.$ TE/TR $\left.=60 / 8210 \mathrm{~ms}, \mathrm{FOV}=224 \times 224 \times 120 \mathrm{~mm}^{3}\right)$ 
with 32 gradient directions $\left(b=1000 \frac{\mathrm{s}}{\mathrm{mm}^{2}}\right)$. The nominal voxel size at acquisition $\left(2 \times 2 \times 2 \mathrm{~mm}^{3}\right)$ was interpolated to $0.875 \times 0.875 \times 2 \mathrm{~mm}^{3}$ after reconstruction.

\section{B. Data processing and fiber-bundle extraction}

Diffusion data obtained at each time-point $(1, \ldots, 8)$ are processed in order to compute the diffusion model using the FDT module of FSL [8]. The obtained images are then used to generate a subject-specific atlas. The atlas is used to time points to the Illinois Institute of Technology (IIT) atlas [19] using DTI ToolKit (DTI-TK) [22]. When each time-point is co-registered to the Atlas, the fractional anisotropy (FA) map is computed in each time-point. Probabilistic streamline tractography is performed using MRTrix [17] based on the fiber orientation density (FOD) information of IIT Atlas. The fiber-bundle extraction is performed using a semi-automatic algorithm [14] coupled with the prior knowledge extracted from the JHU WM fiber-bundle atlas [6].

Formally, an extracted fiber-bundle could be represented as a set $Z=\left\{z_{1}, z_{2}, \ldots, z_{N}\right\}$ composed of $N$ fibers $z_{i}=$ $\left\{\boldsymbol{p}_{\mathbf{1}}, \ldots, \boldsymbol{p}_{\boldsymbol{c}}\right\}$ where $\boldsymbol{p}_{\boldsymbol{q}}=\left(x_{q}, y_{q}, z_{q}\right) \mid 1 \leq q \leq c$. As final post-processing step each fiber is resampled with the same number of points $c=100$ (also called nodes). The coordinate $\boldsymbol{p}_{\boldsymbol{q}}$ is used to extract the voxel's value of the FA maps in the corresponding location of the fiber $z_{i}$ in a specific time-point. With this formalization it is possible to analyze the changes of each diffusion map along each fiber belonging to a bundle.

\section{Blind source separation formalization}

In this work we start from the assumption that the FA signal along each fiber, within a bundle, can be modelled according to the following equation:

$$
f(t)=\sum_{i=1}^{C} \alpha_{i} \sin \left(t \beta_{i}\right)
$$

We use FA instead of other DTI features since its values are in the range $[0 ; 1]$. For our method, we selected $C=$ 10 to model our data. According to this formalization it is possible to reformulate the longitudinal data along a fiberbundle as follows. Let $F^{1}, \ldots, F^{p}$ be the longitudinal FA values along all the fibers within a bundle $F$ from timepoint 1 to time point $P . F^{p}=\left\{f_{1}^{p}(t), \ldots, f_{V}^{p}(t)\right\}$ where each $f_{i}^{p}(t)$ represents the signal at time-point $p$ along the $i-t h$ fiber within the bundle $F$.

Since we are interested in the extraction of the sources containing "pathological" longitudinal changes, we can formalize the problem as a blind source separation (BSS) problem. Give a set of signals $\boldsymbol{X}=\mathbb{R}^{K \times N}$ a BSS problem consists in the extraction of the mixing matrix $M \in \mathbb{R}^{K \times R}$ and/or the original sources in $S \in \mathbb{R}^{R \times N}$ according to the following linear model:

$$
X=M S
$$

where $K$ is the number of observed signals, $R$ is the number of source signals and $N$ is the number of samples per signal.
In our case, the FA signal along each fiber within the bundle is collected in the signal matrix $X$ with $N=P * V$. Each row of the matrix represents the FA signal along a particular fiber in a specific time-point.

\section{Tensorization}

Generally speaking, a $N^{t h}-$ order tensor is an element of the tensor product of $N$ vector spaces, each of which has its own coordinate system; for instance, a third-order tensor has three indices. A first-order tensor is a vector, a second-order tensor is a matrix, and tensors of order three or higher are called higher-order tensors.

In order to describe the tensor-based formalism herein employed, we introduce next the notation used; in particular, if not clearly stated, we use the notation described in [9]. We denote scalar values with small letters (e.g., a), 1-dimensional vectors with bold small letters (e.g., a), matrices with boldface capital letters (e.g., $\boldsymbol{A}$ ) and tensors with boldface Euler script letters (e.g., $\mathcal{A})$.

Consider the signal along the $i-t h$ fiber, belonging to a bundle, described by equation 1 , it can be mapped in an Hankel matrix $H \in \mathbb{R}^{I \times J}$ with $I, J>2 \times C$ defined as follows:

$$
\boldsymbol{H}=\left[\begin{array}{cccc}
f(0) & f(1) & f(2) & \ldots \\
f(1) & f(2) & f(3) & \ldots \\
f(2) & f(3) & f(4) & \ldots \\
\vdots & \vdots & \vdots &
\end{array}\right]
$$

Such representation is in a very compact way a low-rank approximation and it is useful for tensor representation [1].

Given a set of longitudinal signals $F^{1}, \ldots, F^{p}$ of a fiberbundle each composed by a set of functions $f_{i}(t) 1 \leq i \leq V$ it is possible to build a tensor defined as follows. Let $\mathcal{T} \in$ $\mathbb{R}^{I \times J \times N}$, with $N=P * V$, be the tensorized version of the fiber bundle we have:

$$
\mathcal{T}_{[:,:, k]}=H_{\boldsymbol{l}}^{\boldsymbol{g}} \forall 1 \leq k \leq P * V
$$

where $\boldsymbol{H}_{\boldsymbol{l}}^{\boldsymbol{g}} \in \mathbb{R}^{I \times J}$ represents the hankel matrix generated from the function $f_{l}^{g}(t)$.

Roughly speaking, the Hankel matrices generated from the longitudinal acquisition along each bundle are stacked in the $3^{\text {rd }}$ mode of the tensor $\mathcal{T}$. A graphical representation of our tensorization process is described in Figure 1.

Using this procedure, the matrix $X$ of the BSS problem described in equation 2 can be solved in its tensorized version.

\section{E. Canonical polyadic and block term decomposition}

Tensor factorization (decomposition) is a widely used method to identify correlations and relations among different modes of high dimensional data, which finds its application in many research fields such as psychometrics, chemometrics, signal processing, numerical linear algebra, computer vision, numerical analysis, data mining, neuroscience, graph analysis, and elsewhere. The tensor decomposition methods 


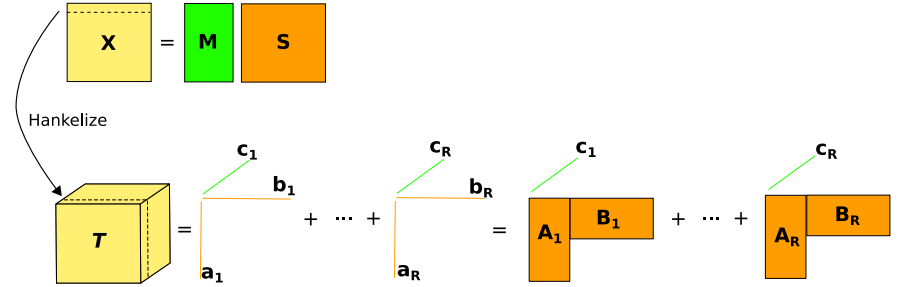

Fig. 1. Tensorization of our problem and factorization using Canonical Polyadic Decomposition (CPD) and $\left(L_{r}, L_{r}, 1\right)$ Block Term Decomposition (BTD).

are mainly generalizations of the Singular Value Decomposition (SVD). In particular, there are numerous factorization methods proposed by researchers such as Higher Order SVD (HOSVD) [10], Tucker decomposition [18], Parallel Factor (a.k.a PARAFAC or CANDECOMP or CP) [5]. The canonical polyadic decomposition (CPD) of the tensor $X \in$ $\mathbb{R}^{c \times m \times n}$ is defined as follows:

$$
\boldsymbol{X}=\sum_{r=1}^{R} \boldsymbol{a}_{\boldsymbol{r}} \circ \boldsymbol{b}_{\boldsymbol{r}} \circ \boldsymbol{c}_{\boldsymbol{r}}+\mathcal{E}
$$

where $R$ is a positive integer, $\boldsymbol{a}_{\boldsymbol{r}} \in \mathbb{R}^{c}, \boldsymbol{b}_{\boldsymbol{r}} \in \mathbb{R}^{m}, \boldsymbol{c}_{\boldsymbol{r}} \in$ $\mathbb{R}^{n} \forall 1 \leq r \leq R$ are the component vectors and $\mathcal{E} \in$ $\mathbb{R}^{c \times m \times n}$ is the error tensor. The symbol " $\circ$ " represents the vector outer product.

The rank of a tensor $\boldsymbol{X}$, denoted $\operatorname{rank}(\boldsymbol{X})$, is defined as the smallest number of rank-one tensors that generate $X$ as their sum. In other words, this is the smallest number of components in an exact CP decomposition, where "exact" means that there is equality in equation 3 with the residual tensor $\mathcal{E}$ as a zero-element tensor. According to the BSS formalization we gave, $\boldsymbol{a}_{\boldsymbol{i}}$ and $\boldsymbol{b}_{\boldsymbol{i}}$ represent the vectors containing information about the sources while each vector $c_{i}$ contains information about the mixtures.

Factorization with rank-1 components performed by CPD could be too restrictive for some applications as it does not model all variability in the data [7]. Using block tensor decomposition (BTD) it is possible to model the variability on the data using a so called rank $\left(L_{r}, L_{r}, 1\right)$ BTD. This decomposition allows to approximates a third-order tensor by a sum of $R$ terms, each of which is an outer product of a $L_{r}-$ rank matrix and a nonzero vector [13]. A $X \in \mathbb{R}^{c \times m \times n}$ can be decomposed by a $\left(L_{r}, L_{r}, 1\right)$ BTD as follows:

$$
X=\sum_{r=1}^{R}\left(A_{r} \cdot B_{r}^{\boldsymbol{T}}\right) \circ c_{r}+\mathcal{E}
$$

The tensor $X$ could be expressed like the sum of the outer products of $L_{r}-$ rank matrices, $\boldsymbol{A}_{\boldsymbol{r}} \in \mathbb{R}^{c \times L_{r}}, \boldsymbol{B}_{\boldsymbol{r}}^{\boldsymbol{T}} \in \mathbb{R}^{L_{r} \times m}$ and the component vector $c_{\boldsymbol{r}} \in \mathbb{R}^{n}$, with $R$ representing the number of components and $\mathcal{E}$ the model error. According to the BSS formalization we gave, $A_{i}$ and $B_{i}$ represent the matrices containing information about the sources while each vector $c_{\boldsymbol{i}}$ contains information about the mixture. Like for the CPD, the value for the rank $R$ and $L_{r}$ should be set a priori. In this study, both CPD and BTD were computed with the unconstrained nonlinear least squares algorithm in the publicly available Tensorlab toolbox [20].

Both CPD and BTD are graphically described in Figure 1 .

\section{F. Fibers differentiation}

Since our goal is to differentiate fibers affected by longitudinal changes and fibers that are not affected, information contained in the factorisation is used. More in detail, we used the information about the mixtures contained in the $c_{\boldsymbol{i}}$ vectors to perform such discrimination. Since the $\boldsymbol{c}_{\boldsymbol{i}}$ vectors have equal size and meaning in both CPD and BTD, the proposed approach is the same for both decomposition algorithms. More in detail, for each fiber $f_{i}$ we build a matrix $\boldsymbol{L}_{\boldsymbol{i}} \in \mathbb{R}^{R \times P} \quad 1 \leq i \leq v$ defined as

$$
\boldsymbol{L}_{\boldsymbol{i}[r,:]}=\boldsymbol{c}_{r[a: b]} \forall 1 \leq r \leq R
$$

where $a=(i-1) * P+1$ and $b=i * P$.

The obtained matrix $\boldsymbol{L}_{\boldsymbol{i}}$ is then used to detect if the $i-t h$ fiber contains (or not) "pathological" longitudinal changes. In order to perform this discrimination, identification of outliers contained in each $\boldsymbol{L}_{\boldsymbol{i}}$ matrix was performed using a densitybased local outliers algorithm (LOF) [2]. This clustering algorithm allows to detect outliers by computing the LOF value for each element. The LOF value of each object represents the degree of the object to be an outlier compared to the other elements. According to this algorithm, we say that a fiber $f_{i}$ is affected by longitudinal changes if there exists a row in its $L_{\boldsymbol{i}}$ matrix labeled as "outlier".

\section{EXPERIMENTS}

Using the data available from the control subject, 60 different "pathological" variations are simulated. All the variations are generated along 3 different fiber-bundles, namely, cortico-spinal tract (CST), superior longitudinal fasciculi (SLF), and inferior longitudinal fasciculi (IFO). Small spherical regions are randomly selected along the WM fiberbundles. Voxels' value inside each of those regions were changed according to the longitudinal simulation paradigm based on generalized Gaussian probability density function proposed in [16]. To quantify the quality of the detection of the fibers containing the simulated variations, the True Positive (TP), True Negative (TN), False Positive (FP) and False Negative (FN) are used to assess the performance. More in detail we focus our attention on the precision $\left(P_{r}=\frac{T P}{T P+F P}\right)$, recall $\left(R_{e}=\frac{T P}{T P+F N}\right)$ and F-Measure $\left(M_{s}=\frac{2 * T P}{2 * T P+F P+F N}\right)$. Since multiple tests are performed, for each of the three diffusion metrics, mean $\left(\overline{P_{r}}, \overline{R_{e}}, \overline{M_{s}}\right)$ and standard deviation $\left(\sigma P_{r}, \sigma R_{e}, \sigma M_{s}\right)$ are computed.

In order to compare the different factorization techniques, tests with CPD and BTD were performed. Moreover, as we discussed in section II-E, values for rank $R$ and $L_{r}$ need to be a-priori known. An important issue in tensor factorization is the estimation of the rank for the CPD factorization of a tensor. The literature on estimating the decomposition rank 
from the tensor is limited. In order to set the value for the rank $R$, we used $R=30$. The best value for the $\left(L_{r}, L_{r}, 1\right)$ rank was selected by performing several tests where different values for $L_{r}$ in the range $\{2,3,4,5\}$ were used.

TABLE I

Changes IN MEAN $\left(\overline{P_{r}}, \overline{R_{e}}, \overline{M_{s}}\right)$ AND STANDARD DEViation $\left(\sigma P_{r}\right.$, $\sigma R_{e}, \sigma M_{s}$ ) IN PARENTHESIS OF PRECISION, RECALL AND F-MEASURE FOR CPD AND BTD. FOR BTD, PERFORMANCES ARE SHOWN ACCORDING TO VARIATION OF THE $\left(L_{r}, L_{r}, 1\right)$ RANK.

\begin{tabular}{|c|ccc|}
\hline & \multicolumn{3}{|c|}{ BTD } \\
\hline $\boldsymbol{L}_{\boldsymbol{r}}$ & $\overline{P_{r}}\left(\sigma P_{r}\right)$ & $\overline{R_{e}}\left(\sigma R_{e}\right)$ & $\overline{M_{s}}\left(\sigma M_{s}\right)$ \\
\hline 2 & $0.55(0.33)$ & $0.33(0.26)$ & $0.35(0.26)$ \\
3 & $0.73(0.06)$ & $\mathbf{0 . 7 0}(\mathbf{0 . 0 6})$ & $\mathbf{0 . 7 1}(\mathbf{0 . 0 4})$ \\
4 & $0.75(0.08)$ & $0.67(0.12)$ & $0.70(0.06)$ \\
5 & $0.80(0.07)$ & $0.56(0.19)$ & $0.63(0.16)$ \\
6 & $\mathbf{0 . 8 3}(\mathbf{0 . 1 1})$ & $0.43(0.20)$ & $0.52(0.16)$ \\
\hline \hline & \multicolumn{3}{|c|}{ CPD } \\
\hline & $0.39(0.27)$ & $0.07(0.4)$ & $0.11(0.09)$ \\
\hline
\end{tabular}
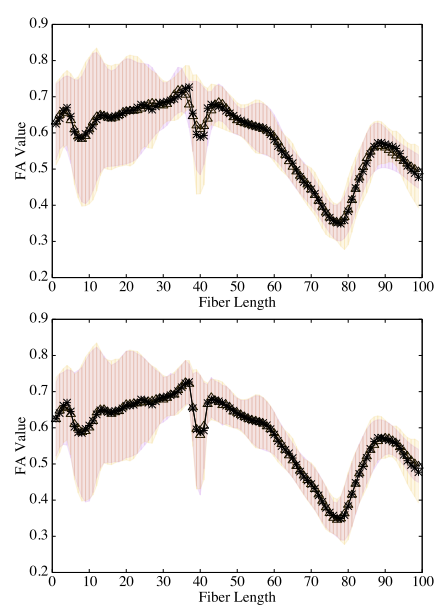

Fig. 2. Mean and standard deviation of the original $(* \pm \quad$ ) and the tensor reconstructed signal $(\triangle \pm \quad$ ) along the cortico-spinal fiber-bundle. Top, graph containing mean and standard deviation of original and CPD fitted signal. Bottom, graph containing mean and standard deviation of original and fitted signal using BTD with $L_{r}=3$.

\section{RESULTS}

We report the results obtained with CPD and BTD for different values of $L_{r}$ in table I (best results are reported in bold). From the tests we performed, clear difference are visible between CPD and BTD. In particular, BTD showed higher performances compared to CPD in terms of Precision, Recall and F-Measure. We also showed how the performances, obtained using the BTD, change according to the $L_{r}$ parameter.

Globally, the best performances were obtained using $L_{r}=$ 3 with a F-Measure of 0.71. Best Precision (0.83) was achieved with $L_{r}=6$ while best Recall (0.71) was achieved with $L_{r}=3$.

By increasing the value of $L_{r}$ it is possible to see an increase of Precision despite Recall. Indeed, with $L_{r} \geq 4$ Precision increases from $0.75\left(L_{r}=4\right)$ to $0.83\left(L_{r}=6\right)$ while Recall decreases from $0.67\left(L_{r}=4\right)$ to $0.43\left(L_{r}=6\right)$.

\section{DISCUSSION}

As expected, BTD gives better results compared to CPD. This is due to the capability of BTD to model, thanks to the $L_{r}-$ rank terms, more variability in the data compared to CPD. This is also confirmed by the performance of the two methods to fit the signal. As shown in figure 2 the overlap between the original signal and the reconstructed signal after factorization, is better when BTD is used compared to CPD. More in detail, in the region containing "pathological changes" ( $x=[38 ; 42])$ the BTD outperforms CPD in the reconstruction of the signal, indeed the overlap between the original and reconstructed signal using BTD is perfect. This better fitting in abnormal regions demonstrates why "pathological changes" are better detected using BTD compared to CPD.

The capability of BTD to better detect those changes could be explained by partial volume effects which affect diffusion images. Partial volume effect originates from the fact that the value of a given voxel is affected by the voxels in its neighbourhood. In case of small pathological changes, partial volume effects can be distinguished to detect such variations. With BTD, thanks to its less restrictive model, it is possible to model such kind of biases present in the data.

\section{CONCLUSIONS}

In this work we described a first application of tensor factorization techniques to longitudinal WM fiber-bundle analysis. At first we describe an easy pipeline to process the data. In the second part we showed how the problem to analyze longitudinal changes along WM fiber-bundles can be formalized as a BSS problem. Finally we described how to tensorize the BSS problem using hankelization and use tensor factorization techniques, namely $\mathrm{CPD}$ and $\mathrm{BTD}$, to perform WM analysis.

As major results we show how tensor-based formalization for BSS is a powerful tool for brain investigation. More in detail, we applied tensor factorization to isolate, from the whole fiber-bundle, just the subset of fibers affected by longitudinal changes.

Furthermore, we enriched our findings showing how different decomposition techniques, namely CPD and BTD, could be applied to the fiber-bundle analysis. Results in term of Precision, Recall and F-Measure were computed on simulated MS data in order to compare how the two factorization techniques are capable to extract "pathological" WM fibers.

In this work, the ability of tensor factorization to study WM was only partially tested. Indeed, we focus our interest just to one single diffusion feature (FA) and to a single modality (DTI). Analysis of multi-parametric information is an interesting application in which tensor based techniques can be further exploited.

As future work we plan to exploit all the information that could be extrapolated for tensor factorization. More in detail, we will study how the other components $\left(\boldsymbol{a}_{\boldsymbol{i}}, \boldsymbol{b}_{\boldsymbol{i}}\right.$ for CPD and $\boldsymbol{A}_{\boldsymbol{i}}, \boldsymbol{B}_{\boldsymbol{i}}$ for BTD) can be used in order to extract useful information about the status of the WM fibers. Moreover, we 
plan to investigate how the use of regularization, like $l_{1}$ or $l_{2}$-norm, on the factorization can improve the performance.

\section{ACKNOWLEDGMENT}

This work is funded by the following projects: EU: MC ITN TRANSACT $2012\left(\mathrm{n}^{\circ}\right.$ 316679). The research leading to these results has received funding from the European Research Council under the European Union's Seventh Framework Programme (FP7/2007-2013) / ERC Advanced Grant: BIOTENSORS ( $\mathrm{n}^{\circ}$ 339804). This paper reflects only the authors' views and the Union is not liable for any use that may be made of the contained information.

\section{REFERENCES}

[1] M. Boussé, O. Debals, L. De Lathauwer, "A Tensor-Based Method for Large-Scale Blind Source Separation using Segmentation," IEEE Transactions on Signal Processing, vol. 65(2), pp. 346-358, 2017.

[2] M.M. Breunig, H.-P. Kriegel, R.T. Ng, J. Sander, "LOF: Identifying density-based local outliers," ACM SIGMOD, vol. 29, pp. 93-104, 2000.

[3] J. B. Colby, L. Soderberg, C. Lebel, I. D. Dinov, P. M. Thompson, E. R. Sowell, "Along-tract statistics allow for enhanced tractography analysis," Neuroimage, vol. 59(4), pp. 3227-3242, 2012.

[4] B. Halandur Nagaraja, D. Sima, N. Sauwen, U. Himmelreich, L. De Lathauwer, S. Van Huffel, "Tensor Based Tumor Tissue Type Differentiation Using Magnetic Resonance Spectroscopic Imaging," Annual International Conference of the IEEE, EMBC, pp. 7003-7006, 2015.

[5] R. A. Harshman, "PARAFAC: Methods of three-way factor analysis and multidimensional scaling according to the principle of proportional profiles," PhD thesis, University of California, Los Angeles, CA, 1976.

[6] K. Hua, J. Zhang, S. Wakana, H. Jiang, X. Li, D. S. Reich, P. A. Calabresi, J. J. Pekar, P. C. M. van Zijl, and S. Mori, "Tract probability maps in stereotaxic spaces: analyses of white matter anatomy and tractspecific quantification," Neuroimage, vol. 39(1), pp. 336-347, 2008.

[7] B. Hunyadi, D. Camps, L. Sorber, W. Paesschen, M. De Vos, S. Van Huffel, L. De Lathauwer, "Block term decomposition for modelling epileptic seizures," EURASIP J. Adv. Signal Process., vol. 139, 2014.

[8] M. Jenkinson, C.F. Beckmann, T.E. Behrens, M.W. Woolrich, S.M. Smith, "FSL," NeuroImage, vol. 62 pp. 782-790, 2012.

[9] T. G. Kolda, B. W. Bader, "Tensor Decompositions and Applications," SIAM Review, vol. 51(3), pp. 455-500, 2009.

[10] L. De Lathauwer, B. D. Moor, J. Vandewalle. "A multilinear singular value decomposition," SIAM J. Matrix Anal. Appl, vol. 21(4), 1253$1278,2000$.

[11] S. Mori, B. J. Crain, V. P. Chacko, P. C. van Zijl, "Three-dimensional tracking of axonal projections in the brain by magnetic resonance imaging," Ann Neurol, vol. 45, pp. 265-269, 1999.

[12] N. Sauwen, D. M. Sima, S. Van Cauter, J. Veraart, A. Leemans, F. Maes, U. Himmelreich, S. Van Huffel, "Hierarchical non-negative matrix factorization to characterize brain tumor heterogeneity using multi-parametric MRI," NMR in Biomedicine, vol. 28(12), pp. 15991624, 2015.

[13] L. Sorber, M. Van Barel, L. De Lathauwer, "Optimization-based algorithms for tensor decompositions: canonical polyadic decomposition, decomposition in rank-() terms, and a new generalization," SIAM J. Optim., vol. 23, pp. 695-720, 2013.

[14] C. Stamile, F. Cauteruccio, G. Terracina, D. Ursino, G. Kocevar, D. Sappey-Marinier, "A Model-Guided String-Based Approach to White Matter Fiber-Bundles Extraction," BIH, LNCS, vol. 9250, pp. 135-144, 2015.

[15] C. Stamile, G. Kocevar, F. Cotton, F. Durand-Dubief, S. Hannoun, C. Frindel, D. Rousseau, D. Sappey-Marinier, "A Sensitive and Automatic White Matter Fiber Tracts Model for Longitudinal Analysis of Diffusion Tensor Images in Multiple Sclerosis," PLoS ONE 11(5): e0156405. doi:10.1371/journal.pone.0156405, 2016.

[16] C. Stamile, G. Kocevar, F. Cotton, F. Maes, D. Sappey-Marinier, S. Van Huffel, "Multiparametric Nonnegative Matrix Factorization for Longitudinal Variations Detection in White-Matter Fiber Bundles," IEEE Journal of Biomedical and Health Informatics, vol. 21(5), pp. 1393-1402, 2017.
[17] J. D. Tournier, F. Calamante, A. Connelly, "MRtrix: Diffusion tractography in crossing fiber regions," International Journal of Imaging Systems and Technology, vol. 22, pp. 53-66, 2012.

[18] L. R. Tucker, "Some mathematical notes on three-mode factor analysis," Psychometrika, vol. 31, pp. 279-311, 1966.

[19] A. Varentsova, S. Zhang, K. Arfanakis, "Development of a high angular resolution diffusion imaging human brain template," Neuroimage, vol. 91, pp. 177-186, 2014.

[20] N. Vervliet, O. Debals, L. Sorber, L. De Lathauwer, "Tensorlab 3.0," Available online, Mar. 2016. URL: http://www.tensorlab.net/.

[21] J. D. Yeatman, R. F. Dougherty, N. J. Myall, B. A. Wandell, and H. M. Feldman, "Tract profiles of white matter properties: automating fiber-tract quantification," PLoS One, vol. 7(11), p. e49790, 2012.

[22] H. Zhang, P. A. Yushkevich, D.C. Alexander, J.C. Gee, "Deformable registration of diffusion tensor MR images with explicit orientation optimization," Medical Image Analysis, vol. 10(5), pp. 764-785, 2006.

[23] R. Zink, B. Hunyadi, S. Van Huffel, M. De Vos, "Tensor-Based Classification of Auditory Mobile BCI without Subject-Specific Calibration Phase," Journal of Neural Engineering, vol. 13(2), 2016. 